\title{
Textile Sensors for the Vehicle Interior
}

Scheibner, Wolfgang; Ullrich, Kay; Neudeck, Andreas; Möhring, Uwe

Textilforschungsinstitut Thüringen-Vogtland e. V. (TITV) Greiz

Zeulenrodaer Str. 42, D-07973 Greiz

\section{Introduction}

Textiles are flexible, breathable and - depending on the application - resistant microstructures, which can be manufactured economically priced in large quantities. Simple components for microsystems like flexible boards, antennas, textile bus systems, sensors and actuators can be produced with new conductive thread materials like SHIELDEX® and ELITEX® [1-3]. This article shows the possibilities of textile sensors as applications for the vehicle interior.

Existing or newly developed textiles can undertake additional tasks in car's interior besides the conventional ones by the use of conductive and sensory fibers. Textile-integrated sensors and their applications are presented based on the operations "conductive“, „sensory“, „protective“, and "decorative“:

1. Textile sensors for load and environmental conditions

2. Textile-integrated energy and data transfer with conductive threads

3. Textile switch and display elements

4. Textiles for passenger monitoring and safety

The presented applications and their enhancements will be discussed under economical and ecological points of view. The most significant advantages here are a reduction in weight, flexibility, breathability of textiles, effective textile processing off the roll, easy assembling in car's interior and individual design with fewer restrictions.

\section{Load sensors (control tear strips)}

Control tear strips can easily indicate an exceeding of load or tension in textiles. One can take the advantage of the control tear strips' mechanical properties, adapted to the particular textile. With correct settings, the control tear strips will break before the textile itself will do. This breaking can be visually recorded, in case the control tear strips are visible on the textile's surface. Electrically or optically conductive sensor threads can also be placed inside the monitored textile, like safety belts, and allow a simple electronically analysis via continuity check.

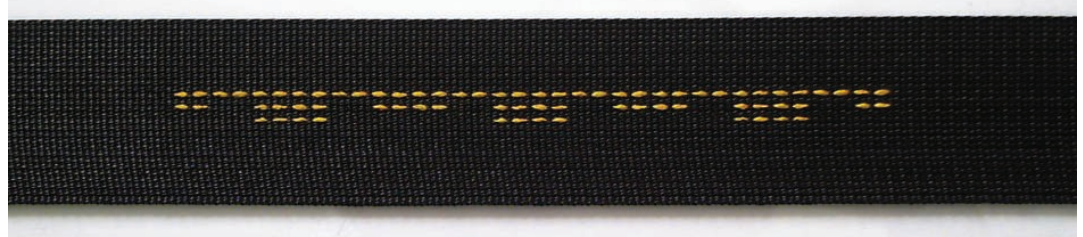

Figure 1: Safety belt with control tear strips for visual evaluation

\section{Tension sensors}

Tension sensors use the change of electrical resistance in a conductive polymer thread as a function of its tension to determine tension values [4]. The tests were made with carbon-black filled silicone threads with a diameter of 0.5 and $1.0 \mathrm{~mm}$. The evaluation of long-term stability of the tension characteristics and the mechanical durability was tested in fast motion laboratory tests. The simulation of load cycles was carried out with a periodic tension of $50 \%$ and subsequent relief. The sensor threads were exposed to 100,000 load changes. Figure 2 shows the example of a tension characteristic for a conductive latex thread. 


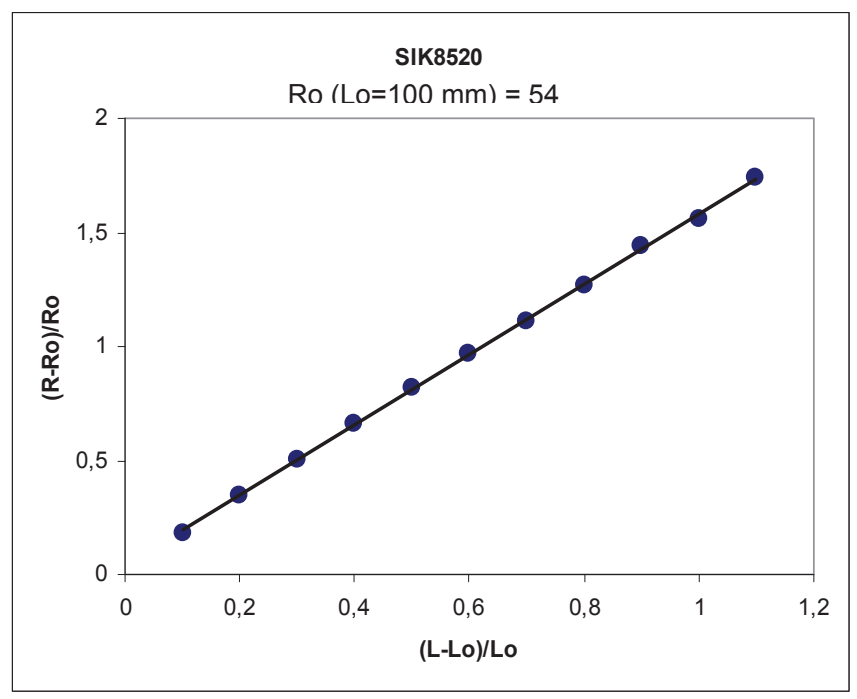

Figure 2: Change of electrical resistance as a function of change of elongation for a conductive silicone latex thread with a diameter of $0.5 \mathrm{~mm}$

Tests for the influence of temperature showed that the electrical resistance for a thread with a diameter of $1.0 \mathrm{~mm}$ is nearly independent from temperature. For a thread with a diameter of $0.5 \mathrm{~mm}$ a temperature coefficient of $0.054 \mathrm{kOhm} / \mathrm{K}$ was determined. The results of cyclic load changes are shown in figure 3 .

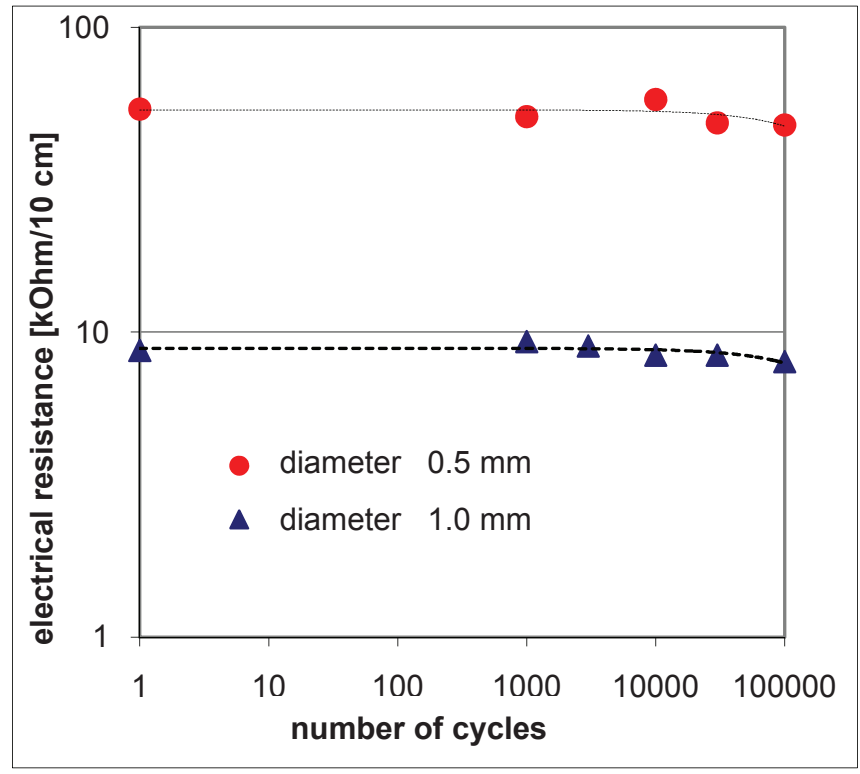

Figure 3: Change of electrical resistance for conductive silicone latex threads after cyclic tension of $50 \%$

\section{Elongation sensor}

An option to conductive latex threads can be realized with conductive thread materials, embroidered on elastic ribbons. Figure 4 shows an example of an embroidered elongation sensor with its ends are embroidered with conductive thread materials. The elastic ribbons were sewed at their ends so that the conductive areas overlap each other and disarrange against each other if the ribbon is stretched. This causes a change of the electrical resistance so that a single value can be attributed to a certain elongation value. The advantages of this layout are a simple adaption of the characteristic through the given geometry of the surface and an easy determination of the elongation with the help of a resistance test.

If a dielectric medium is placed between the two conductive areas which minimizes friction and insulates the areas against each other, a capacitive sensor with the conductive areas working as a condenser is created. The capacity values will be determined by the elongation of the textile. 


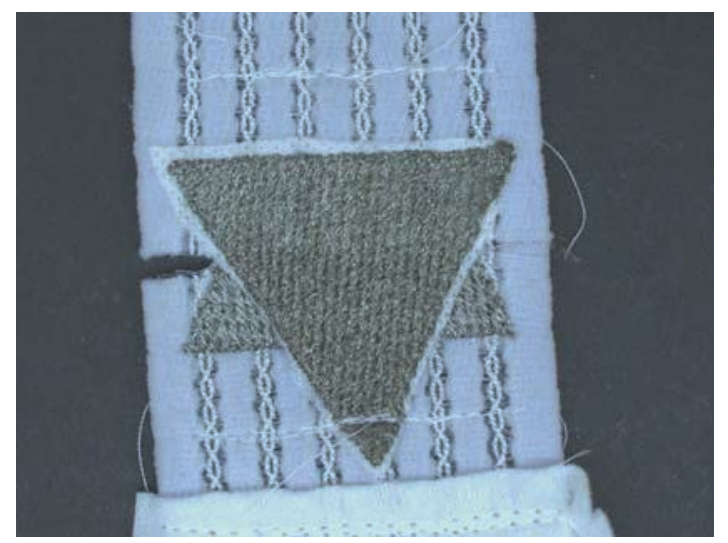

Figure 4: Measuring area of an elongation sensor

Another layout for an elongation sensor consists of an elastic ribbon, where the conductive threads are woven meander-shaped across the tension direction. Because of the low distance between single material webs, statistically distributed contact spots will be created, which deliver a measurable electrical resistance of the whole area. If the ribbon is stretched, the contacts will be pulled apart. The electrical resistance increases. If all contact spots are broken, a constant resistance value is set which is identical with the electrical resistance of the complete fabric. Applications for textile elongation sensors in a car could be safety belts or an occupancy detection for seats.

\section{Pressure and moisture sensors}

Figure 5 shows the characteristic of a force sensor for a conductive thread made of a woven double comb structure with an overlying conductive silicone natural rubber layer $[5,6]$. During the action of force, the resistance of the conductive layer decreases, so that double comb electrode can transfer a forceproportional signal. Because of the change of electrical resistance and the capacity change at the woven electrode with the environmental moisture, a textile conductive or capacitive moisture sensor is generated this way. These sensors made of textile materials could be used as switching elements in a car's seat, as occupancy detection for seats or as the detection of the driver's physical status (skin sensor).

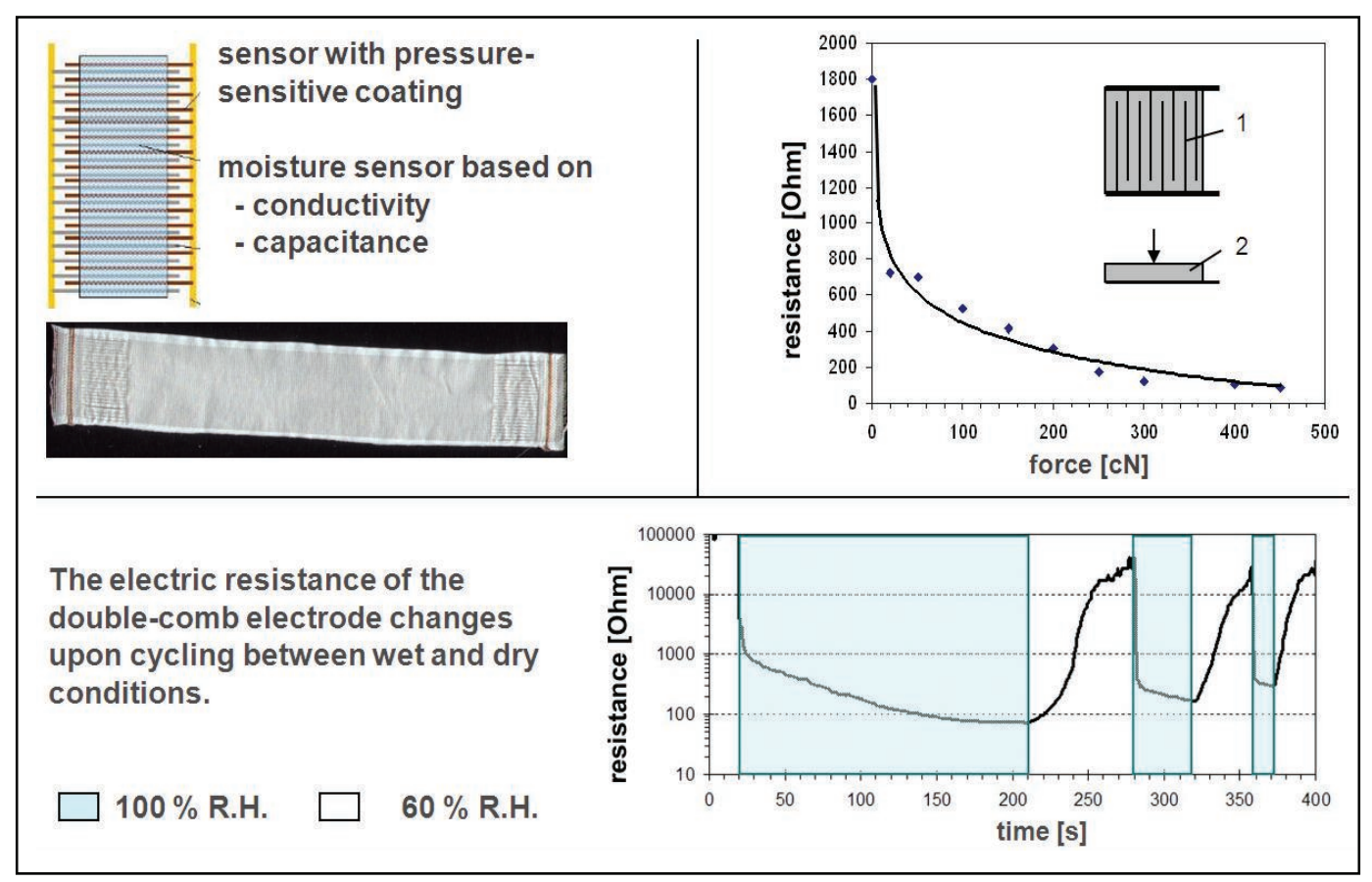

Figure 5: Force-resistance-characteristic for a woven double comb electrode made of conductive threads (1) with a one-sided applied conductive silicone latex layer (2) (up) and moisture dependence of the electrical resistance for the double comb electrode (down) 


\section{Textile-integrated transfer of energy and data [6]}

Electrically conductive textile threads are one of the basic developments for textile sensors as well as for the textile integrated transfer of energy and data. The development of Elitex® threads allows the manufacturing of innovative products or functional components. Elitex ${ }^{\circledR}$ threads are galvanic modified silvered threads made of polyamide. The base material (Shieldex® yarn is provided in different finenesses and has a certain value of conductivity. The resistance depends on the silver layer and goes from $400 \mathrm{Ohm}$ up to $1.5 \mathrm{kOhm}$ per meter. Through an extra galvanic after-treatment process, additional silver is coated on the thread. Depending on the amount of additional silver, the conductivity of the material increases. High electro-plated threads have a resistance from 20 to $40 \mathrm{Ohm}$ per meter. The textile properties of these materials are still given and can be compared with multi filament yarns made of polyester or polyamide. Shedding, twining and enwinding can even increase conductivity and occurs in dependency from the application. So, threads with resistance values of $2 \mathrm{Ohm}$ per meter were processed with the above mentioned base material.

Parallel adjustment of Elitex® threads provides the manufacturing of textile bus structures. Data transfer rates up to $20 \mathrm{Mbit}$ per second are possible. These flexible bus structures are unbeatable with their textile haptic. With very good forming properties, they can be used in places where conventional cables would be difficult to reach. And with attention to a permanent bending load, textile bus structures are durable over a long period. Textile bus structures can be used to realize a textile switch or a textile keyboard.

\section{Textile switching elements $[6,7]$}

Figure 6 shows the layout of a switch, exclusively consisting of textile materials and flexible electric lines. The single switching elements are created made of conductive narrow fabrics, separated by a distancekeeping textile with openings. For this, a spacer fabric with bending resistant pile yarns could be used, whose restoring force defines the responsivity of the switch. A freely configurable matrix of switching elements can be created depending on the desired function. Contacting is realized by applying pressure on a switching element.

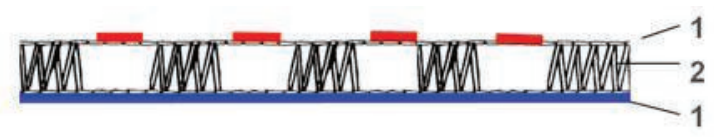

Schematic depiction of a textile switch

1 - tapes from electrically conductive ELITEX fibers

2 - separator (spacer fabric) with openings to allow electrical contact when applying pressure
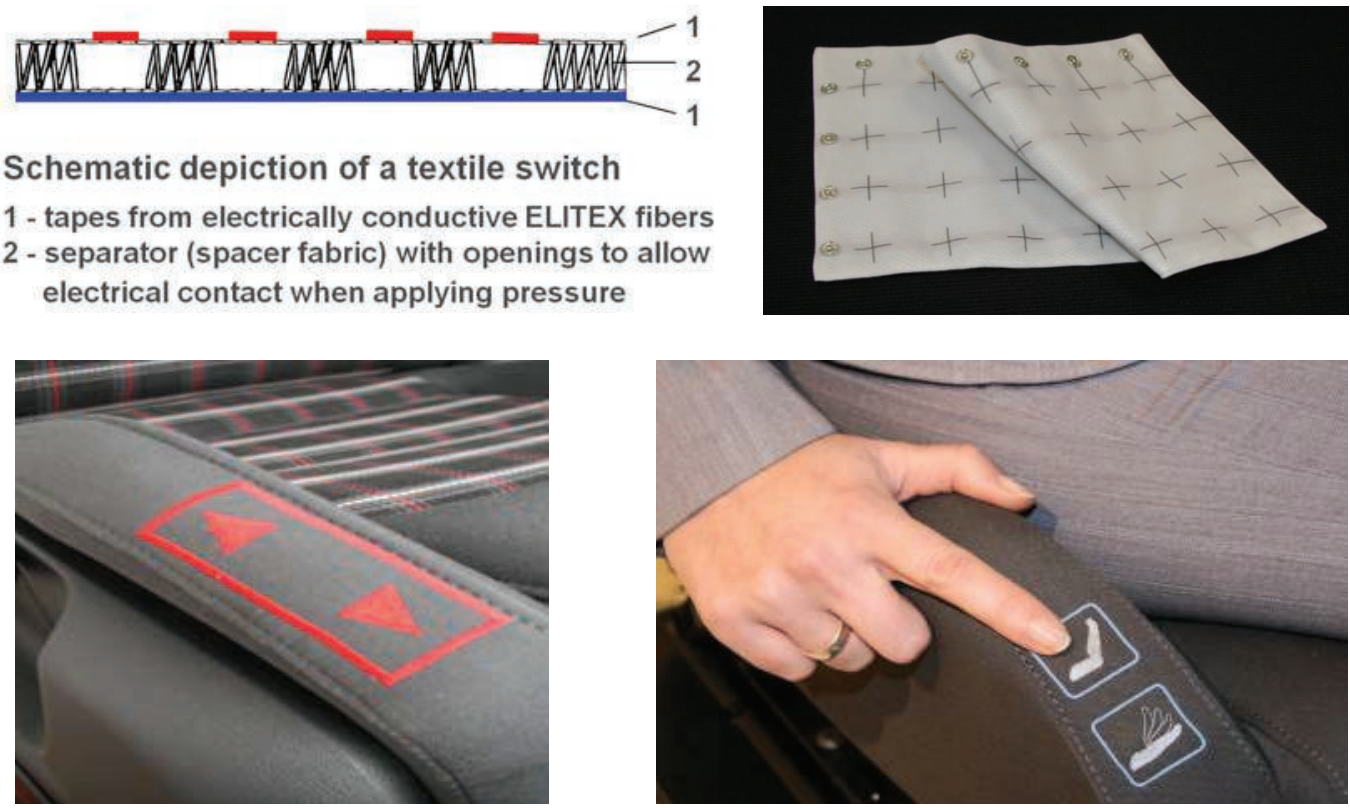

Figure 6: Layout of a textile switch, switching matrix as a mat and textile switching elements on a car's seat 


\section{Literature}

[1] A. Neudeck et al., Technologie zur galvanischen und elektrochemischen Modifizierung von vorstrukturierten partiell leitfähigen textilen Flächen, Unitex, 2 (2006) 12-14.

[2] S. Gimpel et al., Textile-based Electronic Substrate Technology, J. of Industrial Textiles $\underline{33}$ (2004) 3, S. 179

[3] A. Neudeck et al., New Kinds of Yarn Preparation for Medical Applications and to Manufacture Interactive Textile Micro Systems, Proceedings of the 8. Thüringer Grenz- und Oberflächentag, Jena 2008

[4] W. Scheibner et al., Textilien für sensorische und aktuatorische Applikationen, In: GMMFachbericht Nr. 58, VDE-Verlag, Berlin, Offenbach 2009, ISBN 978-3-8007-3146-6

[5] U. Möhring, Textilintegrierte Sensorik für den Autoinnenraum, Symposium mtex 2010, 9. Juni 2010, Chemnitz

[6] U. Möhring et al., Conductive, sensorial and luminescent features in textile structures, In: Proceeding of the $3^{\text {rd }}$ International Forum on Applied Wearable Computing, Bremen 15-16-March 2006, VDE-Verlag, Berlin, Offenbach 2006, ISBN 978-3-8007-2954-8

[7] W. Scheibner et al., Fibers for Smart Textiles - Applications in the Vehicle Interior, Proceedings of the $49^{\text {th }}$ Dornbirn Man-Made Fibers Congress 15-17 September 2010

\section{Acknowledgement}

We would like to thank Federal Ministry of Education and Research for the financial support of the research project "SeatSen" BMBF 16SV3457 which was funded within the framework programme Microsystems (2004 - 2009) and helpfully assisted by VDI/VDE Innovation + Technik GmbH. 